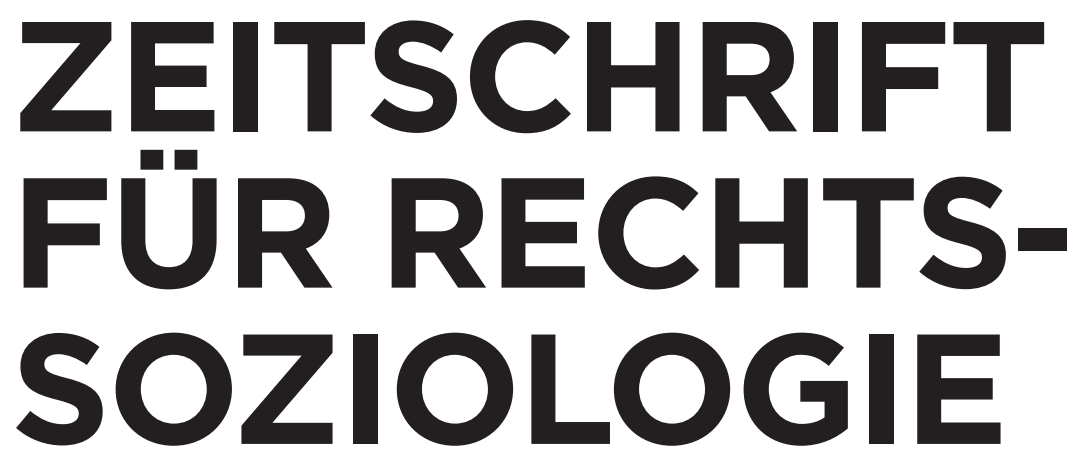

\title{
THE GERMAN JOURNAL OF LAW AND SOCIETY
}

\author{
HERAUSGEGEBEN VON \\ Alfons Bora \\ Christian Boulanger \\ Michelle Cottier \\ Walter Fuchs \\ Pierre Guibentif \\ Vagias Karavas \\ Andrea Kretschmann \\ Stefan Machura \\ Doris Schweitzer
}

\section{DE GRUYTER \\ OLDENBOURG}


ABSTRACTED/INDEXED IN Baidu Scholar · Cabells Journalytics · CNKI Scholar (China National Knowledge Infrastructure) · CNPIEC: cnpLINKer · Dimensions · EBSCO (relevant databases) · EBSCO Discovery Service · Google Scholar · IBR (International Bibliography of Reviews of Scholarly Literature in the Humanities and Social Sciences) · IBZ (International Bibliography of Periodical Literature in the Humanities and Social Sciences) · J-Gate · JournalTOCs · KESLI-NDSL (Korean National Discovery for Science Leaders) · Microsoft Academic · MyScienceWork · Naver Academic · Naviga (Softweco) Primo Central (ExLibris) · Publons · QOAM (Quality Open Access Market) · ReadCube · SCImago (SJR) - SCOPUS · Semantic Scholar · Sherpa/RoMEO · Summon (ProQuest) · TDNet · Ulrich's Periodicals Directory/ulrichsweb · WanFang Data · WorldCat (OCLC) $\cdot$ Yewno Discover

ISSN 0174-0202 · e-ISSN 2366-0392

Alle Informationen zur Zeitschrift, wie Hinweise für Autoren, Open Access, Bezugsbedingungen und Bestellformulare, sind online zu finden unter www.degruyter.com/zfrs

HERAUSGEGEBEN VON Alfons Bora (Bielefeld), Christian Boulanger (Frankfurt), Michelle Cottier (Genf), Walter Fuchs (Wien), Pierre Guibentif (Lissabon), Vagias Karavas (Luzern), Andrea Kretschmann (Lüneburg), Stefan Machura (Bangor, Wales), Doris Schweitzer (Gießen)

REDAKTION Dr. Walter Fuchs, MA, IRKS Institut für Rechts- und Kriminalsoziologie, Museumsstraße 5/12, 1070 Wien, Österreich. E-Mail: walter.fuchs@irks.at / Dr. Christian Boulanger, Max-Planck-Institut für Rechtsgeschichte und Rechtstheorie, Hansaallee 41, 60323 Frankfurt am Main, E-Mail: boulanger@ rg.mpg.de

WISSENSCHAFTLICHER BEIRAT Erhard Blankenburg (Amsterdam) $\uparrow$, Brun-Otto Bryde (Gießen), Kai-D. Bussmann (Halle), Fritz Jost (Bielefeld), Susanne Karstedt (Mt. Gravatt), Wolfhard Kohte (Halle), Rüdiger Lautmann (Bremen), Martin Morlok (Düsseldorf), Konstanze Plett (Bremen), Klaus F. Röhl (Bochum), Ralf Rogowski (Warwick), Gunther Teubner (Frankfurt a. M.), Manfred Weiss (Frankfurt a. M.)

VERLAG Walter de Gruyter GmbH, Berlin/Boston, Genthiner Straße 13, 10785 Berlin, Germany

JOURNAL MANAGER Jana Kuchta, De Gruyter, Genthiner Straße 13, 10785 Berlin, Germany, Tel.: +49 (0)30 260 05-228, Fax: +49 (0)30 260 05-250, E-Mail: jana.kuchta@ degruyter.com

ANZEIGENVERANTWORTLICHE Jana Kuchta, De Gruyter, Genthiner Straße 13, 10785 Berlin, Deutschland, Tel.: +49 (0)30 260 05-170, E-Mail: anzeigen@degruyter.com

(C) 2021 Walter de Gruyter GmbH, Berlin/Boston

SATZ Dörlemann Satz, Lemförde

DRUCK Franz X. Stückle Druck und Verlag e.K., Ettenheim

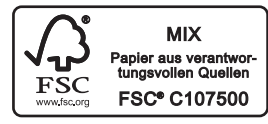




\section{Inhalt}

\section{Strafrecht und neue Formen sozialer Kontrolle}

Tobias Singelnstein

Einleitung zum Themenschwerpunkt —1

Andrea Kretschmann und Aldo Legnaro

Die „drohende Gefahr“ als Schlüsselbegriff einer Sekuritisierung des

Rechts - 3

Simon Egbert

Predictive Policing als Treiber rechtlicher Innovation? — 26

Julia Habermann und Tobias Singelnstein

Sanktionierungsvorstellungen und Einstellungen des deutschen

Justizpersonals im Strafrecht -52

Christine Morgenstern

Need for Speed: Die neue Beschleunigungsfreude im Strafverfahren am

Beispiel der Untersuchungshaft — 90

\section{Abhandlungen}

Gunther Teubner

Die Verfassung gesellschaftlicher Mehrwerte — 117

Minou Banafsche und Tanja Klenk

Die Verwaltungspraxis in der Grundsicherung für Arbeitsuchende - eine rechtstatsächliche Analyse am Beispiel der kommunalen Jobcenter —151

Anja Böning, Frerk Blome und Christina Möller

Vom kollektiven zum individualisierten Aufstieg? — 179

Mirostaw Michat Sadowski

City as a Locus of Collective Memory. Streets, Monuments and Human Rights 209 


\section{Dokumentation}

Michelle Cottier

Zur rechtssoziologischen Vermessung der Abschaffung, Transformation und Entformalisierung von Recht -241

\section{Rezensionen}

\section{Clemens Boehncke}

Auch eine Geschichte des Rechts. Rezension von Nils Jansen, Recht und gesellschaftliche Differenzierung - Fünf Studien zur Genese des Rechts und seiner Wissenschaft $\longrightarrow 252$

\section{Claus Gienke}

Matthias Mahlmann, Widerständige Gerechtigkeit. Der Angriff auf Demokratie, Verfassungsstaat und Menschenrecht und die Gesellschaftstheorie des Rechts -263

\section{Lisa Hahn}

Alexander Graser \& Helmrich, Christian, Strategic Litigation. Begriff und Praxis 270

\section{Klaus Schlichte}

Larissa Vetters, Prozesse des State Building in Bosnien-Herzegowina. Eine Verwaltungsethnographie -277

Ulrike Schultz

Anna Lena Göttsche, Weibliche Genitalverstümmelung/Beschneidung. Interdisziplinäre Betrachtungen und rechtliche Einordnungen im Lichte von Grund- und Menschen rechten - 280 